\title{
ESCOLARIDADE FEMININA E A SUA IMPORTÂNCIA NA ESCOLHA VOLUNTÁRIA DA FECUNDIDADE
}

\author{
LA ESCOLARIZACIÓN FEMENINA Y SU IMPORTANCIA EN LA ELECCIÓN \\ VOLUNTARIA DE LA FERTILIDAD
}

\section{FEMALE SCHOOLING AND ITS IMPORTANCE IN THE VOLUNTARY CHOICE OF FERTILITY}

Natalia DUS POIATTI ${ }^{1}$

RESUMO: A Conferência Internacional sobre População e Desenvolvimento das Nações Unidas (CIPD) de 1994 identificou a centralidade da defesa dos direitos reprodutivos e a importância de se garantir a realização de escolhas reprodutivas voluntárias (UNFPA, 2019). O presente artigo apresenta uma revisão sobre os principais modelos teóricos de demanda por filhos e, avaliando a contrapartida empírica desses modelos em uma base de dados internacional, aponta as principais variáveis socioeconômicas determinantes da fecundidade. Esse estudo mostra que a escolaridade feminina é a variável socioeconômica mais importante para explicar o número de filhos por mulher, visto que o aumento da educação feminina pode expandir o acesso à informação sobre os métodos contraceptivos, melhorar as oportunidades de emprego e "empoderar" as mulheres na defesa de suas escolhas sexuais e reprodutivas.

PALAVRAS-CHAVE: Fecundidade. Educação. Desigualdade de gênero. Políticas populacionais. Desenvolvimento socioeconômico.

RESUMEN: La Conferencia Internacional de las Naciones Unidas sobre Población y Desarrollo (CIPD) de 1994 identificó la centralidad de defender los derechos reproductivos y la importancia de garantizar opciones reproductivas voluntarias (UNFPA 2019). Este artículo presenta una revisión de los principales modelos teóricos de demanda de niños y, al evaluar la contraparte empírica de estos modelos en una base de datos internacional, señala las principales variables socioeconómicas que determinan la fertilidad. Este estudio muestra que la escolarización femenina es la variable socioeconómica más importante para explicar el número de hijos por mujer, ya que una mayor educación femenina puede ampliar el acceso a la información sobre métodos anticonceptivos, mejorar las oportunidades de empleo y "empoderar" a las mujeres en defensa de sus elecciones sexuales y reproductivas.

PALABRAS CLAVE: Fecundidad. Educación. Desigualdad de género. Políticas de población. Desarrollo socioeconómico.

ABSTRACT: The 1994 United Nations International Conference on Population and Development (ICPD) identified the centrality of defending reproductive rights and the

1 Instituto de Relações Internacionais da Universidade de São Paulo (IRI-USP), São Paulo - SP - Brasil. Docente. Doutorado em Economia Financeira pela London Business School (LBS). ORCID: http://orcid.org/0000-0001-9660-8544.E-mail: npoiatti@usp.br 
importance of ensuring voluntary reproductive choices (UNFPA, 2019). This paper presents a review of the main theoretical models of demand for children and, by assessing the empirical counterpart of these models in an international database, points out the main socioeconomic variables determining fertility. This study shows that female schooling is the most important socioeconomic variable to explain the number of children per woman, as increased female education can expand access to information on contraceptive methods, improve employment opportunities and "empower" women in defending their sexual and reproductive choices.

KEYWORDS: Fecundity. Education. Gender inequality. Population policies. Socioeconomic development.

\section{Introdução}

A Conferência Internacional sobre População e Desenvolvimento das Nações Unidas (CIPD) de 1994 identificou a centralidade da defesa e promoção dos direitos reprodutivos e da igualdade de gênero para melhorar o desenvolvimento socioeconômico. Naquele momento, diversos grupos feministas e defensores dos direitos reprodutivos lutavam contra as políticas de controle populacional que alguns países adotaram nas décadas de 1970 e 1980 para limitar o tamanho das famílias compulsoriamente (UNFPA, 2019). De acordo com o programa de ações proposto na CIPD, seria necessário dar acesso e informar os casais sobre métodos contraceptivos modernos, além de "empoderar" as mulheres, possibilitando que elas pudessem fazer as suas escolhas reprodutivas voluntariamente.

Diferentes regiões do mundo apresentaram suas transições demográficas para menores níveis médios de fecundidade em diferentes períodos. Nos países de mais alta renda, as taxas de fecundidade total caíram de aproximadamente 2,8 crianças por mulher em 1950 para 1,9 em 1990 (SCHULTZ, 1997). No Brasil, o declínio da fecundidade entrou em sua fase mais acelerada em meados da década de 1960, fato este intimamente relacionado a mudanças socioeconômicas e culturais. Entretanto, esse declínio não ocorreu de forma uniforme para todas as classes de renda, situação educacional, e grau de urbanização domiciliar. Como evidencia Berquó e Cavenaghi (2014), os domicílios brasileiros com menores níveis de renda e educação ainda apresentam taxas de fecundidade relativamente altas.

De acordo com Blake (1981), altas taxas de fecundidade em determinados segmentos da população podem representar um problema social, visto que crianças nascidas em famílias numerosas têm maior probabilidade de sofrer diversos problemas de saúde na infância. Um maior número de filhos por mulher pode levar a uma redução do peso médio ao nascer e a 
uma maior taxa de mortalidade infantil. Para melhorar a qualidade de vida da população, torna-se importante entender os principais determinantes da fecundidade.

Empiricamente, a transição para níveis menores de fecundidade foi concomitante ao aumento do desenvolvimento econômico em diferentes regiões do mundo. Bryant (2007) apresenta evidências empíricas da associação negativa entre níveis de fecundidade e desenvolvimento socioeconômico. Até 2015, o governo chinês estabeleceu a política do filho único, penalizando severamente casais com mais de um filho. Apesar do caráter coercitivo dessa política, há evidência de que a maior parte da queda da fecundidade chinesa após 1980 foi causada pelo desenvolvimento socioeconômico do país, caracterizado por uma melhoria no poder aquisitivo da população e no acesso a serviços de educação (ZHANG, 2017).

Utilizando dados do censo demográfico brasileiro, Araújo Junior, Salvato e Queiroz (2013) mostram que um maior desenvolvimento socioeconômico, medido pelo índice de desenvolvimento humano (IDH), estaria associado a patamares menores de fecundidade. De fato, os níveis de educação, de renda e o acesso a serviços de saúde, os quais compõem o IDH, foram apontadas na literatura como possíveis variáveis explicativas na determinação do número de filhos por mulher. No Brasil, Brandão (2006) enfatiza a disponibilidade de métodos contraceptivos modernos em larga escala e, não obstante, altas taxas de gravidez indesejada. Além do acesso a métodos de contracepção, o presente estudo indica a necessidade de um aumento da educação feminina, a fim de que as mulheres consigam buscar e interpretar as informações sobre os métodos disponíveis.

A figura 1 mostra a taxa de fecundidade total, por ano, segundo a escolaridade. O eixo vertical representa a média da taxa de fecundidade total, dada pelo número médio de crianças por mulher, para quatro grupos de mulheres de acordo com os seus níveis de escolaridade: de 0 a 3 anos, de 4 a 7 anos, de 8 a 10 anos e mais de 10 anos. 
Figura 1 - Gráfico da Taxa de Fecundidade Total, segundo a Escolaridade

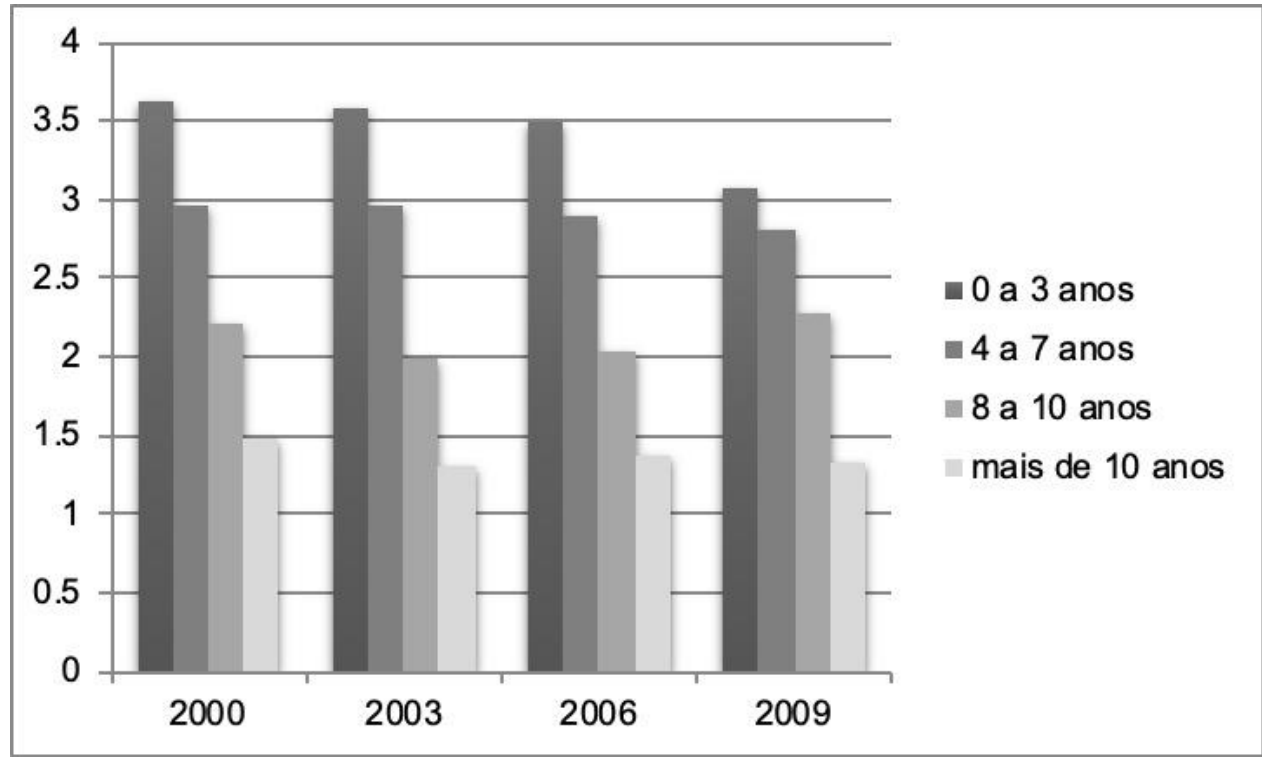

Fonte: CEPI-DSS/ENSP/FIOCRUZ (2012)

Apesar da taxa média de fecundidade total brasileira permanecer em torno da taxa de reposição da população durante todo o período, declinando de 2,34 em 2000 para 1,94 em 2009, as taxas médias para as mulheres com até 7 anos de estudo ainda permanecem próximas de 3 filhos por mulher. Como ilustra o gráfico, há uma associação negativa entre fecundidade e nível educacional feminino: quanto maior o número de anos de estudo, menor o nível de fecundidade.

Entretanto, não se pode estabelecer uma relação de causalidade entre nível educacional feminino e fecundidade com base nessa evidência empírica. $\mathrm{O}$ acesso a serviços de educação e saúde está também relacionado ao nível de renda da população, levando à movimentação conjunta dessas variáveis ao longo do tempo. De fato, Berquó e Cavenaghi (2014) registram a associação negativa entre rendimento domiciliar per capita e fecundidade no Brasil. De acordo com esse estudo, municípios com rendimento domiciliar per capita de até um quarto de salário mínimo ainda apresentavam um número médio de 4,1 filhos em 2010.

O presente estudo pretende apresentar uma revisão sobre os principais modelos teóricos de demanda por filho. Além disso, visa estimar a contrapartida empírica desses modelos em uma base de dados internacional, e avaliar os principais fatores socioeconômicos que explicam a fecundidade, distinguindo os impactos de cada um dos fatores componentes do índice de desenvolvimento humano: educação, saúde e renda. O entendimento dos fatores que impactam a fecundidade é essencial para que as mulheres possam ter maior controle sobre o número de filhos e exercer as suas escolhas reprodutivas ideais. 
A primeira seção contém uma revisão teórica sobre os principais determinantes da fecundidade. A segunda seção utiliza o arcabouço teórico de demanda por filhos apresentado na primeira seção e apresenta uma análise empírica sobre os principais determinantes da fecundidade. A terceira seção descreve os principais resultados da análise empírica, e a quarta seção apresenta as principais conclusões e indicações para pesquisas futuras.

\section{Modelos teóricos da fecundidade}

Desde a década de 1960, um grupo de pesquisadores tem analisado o comportamento reprodutivo da família, considerando esta como uma importante entidade alocadora de seus escassos recursos a fim de atender seus propósitos de consumo corrente e investimento em filhos.

Os modelos desenvolvidos, liderados por Becker (1960) e aprimorados por Schultz (1997), são orientados para o estudo da escolha do número de filhos e para a alocação de tempo nos domicílios. Eles mostram que o comportamento dos domicílios responde a mudanças na tecnologia, na restrição orçamentária e nos gostos.

De acordo com esses modelos teóricos da fecundidade, o aumento do nível educacional de um indivíduo em idade reprodutiva melhora as suas oportunidades salariais no mercado de trabalho. Isto é de extrema importância para as mulheres, visto que as mães, em média, ainda dedicam mais tempo do que os pais no cuidado dos filhos, e ficam impossibilitadas de exercer algumas atividades no mercado de trabalho. Dessa forma, o aumento do nível médio educacional feminino está associado ao aumento do salário médio feminino e do custo de oportunidade de cuidado dos filhos, reduzindo a fecundidade. Da mesma forma, quanto maior a participação feminina no mercado de trabalho, menor o tempo disponível para o cuidado dos filhos e menor o nível de fecundidade.

De maneira análoga, o aumento médio da educação masculina pode reduzir a fecundidade, se o tempo que os homens investem no cuidado dos filhos os impedem de exercer algumas atividades no mercado de trabalho, ou pode aumentar a fecundidade, se o aumento da renda salarial devido ao maior nível educacional possibilitar o dispêndio no cuidado de filhos adicionais.

Após o controle das variáveis de educação, elevações na renda implicarão em aumento da quantidade de recursos financeiros para se dispender no cuidado dos filhos e criarão um efeito renda na fecundidade, o qual é assumido ser positivo. 
Ademais, a demanda dos pais por nascimentos pode ser vista como uma demanda derivada do valor que os pais dão às crianças sobreviventes. Uma redução da mortalidade infantil reduz o custo de se produzir um sobrevivente, porém reduz o número necessário de nascimentos para se ter um sobrevivente. Nesses modelos, a redução da mortalidade infantil é associada com o declínio na demanda por nascimentos.

A pesquisa na área é extensa, o que demonstra a relevância do problema e a necessidade de ampliar nosso entendimento sobre as variáveis que impactam os padrões de fecundidade.

\section{Análise empírica sobre os determinantes da fecundidade}

Nessa seção, realizaremos uma análise empírica sobre os determinantes da fecundidade em uma base de dados internacionais. O modelo econométrico estimado relaciona taxa de fecundidade total às variáveis explicativas apontadas pelo modelo teórico de demanda por filhos de Schultz (1997). O banco de dados está organizado no formato painel e contém dados a partir da década de 1960, de 5 em 5 anos, para todos os países para os quais havia disponibilidade de dados e explicitados na Figura 2.

Figura 2 - Amostra da pesquisa: países analisados

\begin{tabular}{|l|l|l|l|l|l|l|}
\hline África do Sul & Canadá & Filipinas & Indonésia & Mali & Reino Unido & Turquia \\
\hline Alemanha & Chile & Finlândia & Irã & Mauritânia & R. Dominicana & Uganda \\
\hline Algéria & China & França & Irlanda & México & Ruanda & Uruguai \\
\hline Argentina & Chipre & Gambia & Israel & Moçambique & Senegal & Venezuela \\
\hline Austrália & Cingapura & Ghana & Itália & Nepal & Serra Leoa & Iêmen \\
\hline Áustria & Colômbia & Grécia & Islândia & Nova Zelândia & Sri Lanka & Zâmbia \\
\hline Bahrain & Congo & Guatemala & Jamaica & Nicarágua & Sudão & Zimbábue \\
\hline Bangladesh & Costa Rica & Guiné-Bissau & Japão & Nigéria & Swaziland & \\
\hline Barbados & Dinamarca & Guiana & Jordânia & Noruega & Suécia & \\
\hline Bélgica & Equador & Haiti & Kenya & Paquistão & Suíca & \\
\hline Benin & Egito & Holanda & Korea & Panamá & Síria & \\
\hline Bolívia & El Salvador & Honduras & Kuwait & Papua N. Guiné & Tanzânia & \\
\hline Botsuana & Em. Árabes & Hong Kong & Lesoto & Paraguai & Tailândia & \\
\hline Brasil & Espanha & Hungria & Libéria & Peru & Togo & \\
\hline Burundi & EUA & I. Maurício & Malaui & Polônia & Trinidad e Tobago \\
\hline Camarões & Fiji & Índia & Malásia & Portugal & Tunísia & \\
\hline
\end{tabular}

Fonte: elaborada pelo autor

A equação abaixo (Figura 3) representa o modelo de regressão proposto:

Figura 3 - Modelo de regressão proposto

$$
Y_{i t}=c+\sum_{k=1}^{7} b_{k} X_{k i t}+a_{i}+u_{i t}
$$

Fonte: elaborada pelo autor 
Em que:

"Y" equivale à taxa de fecundidade total proveniente do Banco Mundial (2015) e representa o número médio de crianças por mulher ao longo de sua vida reprodutiva;

"X" é uma matriz constituída por cinco variáveis explicativas do Banco Mundial (2015): 1. PIB per capita em dólares americanos constantes, 2. taxa de mortalidade infantil, 3. expectativa de vida, 4. proporção da população urbana, 5. taxa de participação feminina na população economicamente ativa; bem como duas variáveis provenientes de Barro-Lee (2013): 6. média de anos de estudo feminino e 7. média de anos de estudo masculino.

“a” representa a variável de efeito-fixo ou não-observável por país; "u” representa o erro idiossincrático; "b" consiste na matriz de parâmetros a ser estimada; "c" representa uma constante; o subscrito “i” identifica os países, o subscrito "t", os anos, e "k", as sete variáveis explicativas.

Uma possível forma de estimação seria a realização de um 'pool' por mínimos quadrados ordinários. Entretanto, para que a matriz de parâmetros "b" seja consistentemente estimada, o erro " $v=a+u$ " não pode ser correlacionado com " $X$ ". Mesmo que " $u$ " seja não correlacionado com as variáveis explicativas, o mesmo não se pode ser dizer sobre a variável de efeito-fixo. Especificamente para se explicar a fecundidade, variáveis geográficas, culturais e históricas, distintas para cada país e constantes no tempo, provavelmente estão correlacionadas com as variáveis explicativas utilizadas na estimação. Este é o caso, por exemplo, da variável religião predominante em cada país, que, possivelmente, é correlacionada com a renda per capita. Nesse caso, a estimação dos parâmetros pelo método de mínimos quadrados ordinários seria inconsistente (ARELLANO, 2003). A organização dos dados no formato painel permite que essa correlação exista e que o viés na estimação dos parâmetros seja eliminado, através da utilização do estimador de efeito-fixo.

Mesmo após o controle do efeito-fixo por país, há grande probabilidade de presença de endogeneidade no modelo, ou seja, de existência de correlação entre as variáveis explicativas e o erro idiossincrático. Por exemplo, Sander (1992) indica a provável endogeneidade da variável educação feminina, a qual seria não somente variável explicativa como também explicada pelo número de filhos por mulher. $\mathrm{O}$ mesmo pode-se dizer sobre a participação feminina na força de trabalho. Mulheres com um maior número de filhos provavelmente alocam uma maior parte do tempo para o cuidado das crianças em detrimento dos seus estudos e de sua participação na força de trabalho. Em adição, mulheres com maior taxa de fecundidade também podem investir tempo e recursos monetários no cuidado de cada um de seus filhos, o que pode aumentar a taxa de mortalidade infantil. O procedimento econométrico 
utilizado para lidar com esses problemas é o estimador de Arellano-Bond (ARELLANO, 2003) pelo método dos momentos generalizado, em que variáveis explicativas defasadas e variáveis diferenciadas defasadas são utilizadas como instrumentos para as variáveis explicativas endógenas: participação feminina na força de trabalho, média de anos de estudo feminino e taxa de mortalidade infantil.

\section{Discutindo os determinantes da fecundidade}

A Figura 3 reporta os impactos de cada variável explicativa na fecundidade para o modelo de efeito-fixo por país. A primeira coluna contém as variáveis explicativas, a segunda e a terceira, os impactos estimados $b_{1} a b_{7}$ e os seus desvios-padrão entre parênteses (considerando-se erro-padrão robusto Huber-White) para os modelos estático e dinâmico, respectivamente.

Figura 3 - Tabela de Determinantes da Fecundidade e seus respectivos Impactos

\begin{tabular}{lcl}
\hline Variáveis Explicativas & Modelo Estático & Modelo Dinâmico \\
\hline Fecundidade (t-1) & --------- & $+1,09(0,01)^{*}$ \\
Feundidade (t-2) & -------- & $-0,42(0,01)^{*}$ \\
Escolaridade Feminina & $-0,56(0,09)^{*}$ & $-0,37(0,02)^{*}$ \\
Participação Feminina (\%) & $-0,05(0,01)^{*}$ & $+0,01(0,00)^{*}$ \\
Escolaridade Masculina & $+0,22(0,08)^{*}$ & $+0,21(0,01)^{*}$ \\
Mortalidade Infantil & $+0,01(0,00)^{*}$ & $+0,01(0,00)^{*}$ \\
Expectativa de Vida & $-0,02(0,02)$ & $+0,01(0,00)^{*}$ \\
População Urbana (\%) & $-0,01(0,01)$ & $-0,00(0,00)^{*}$ \\
Pib per capita & $+0,00(0,00)^{*}$ & $+0,00(0,00)^{*}$ \\
\hline N observações & $\mathbf{9 2 4}$ & $\mathbf{6 1 7}$ \\
R-quadrado & $\mathbf{0 , 7 8}$ & $\mathbf{0 , 8 6}$ \\
\hline
\end{tabular}

*Variáveis estatisticamente significantes ao nível de 5\%; 2. O teste Hausman apontou para a inconsistência do método de efeito-aleatório.

Fonte: elaborada pelo autor

De acordo com o modelo teórico da fecundidade, o aumento da educação feminina está associado a ganhos de produtividade da mulher no mercado de trabalho, o que aumenta o custo do cuidado dos filhos e reduz a fecundidade. Conforme esperado pela teoria, após o controle das demais variáveis explicativas, anos médios de educação feminina tem um impacto negativo e significante na taxa de fecundidade no modelo estimado (Figura 3).

Mais especificamente, o aumento de um ano médio de educação feminina ocasiona uma queda de aproximadamente 0,6 filhos por mulher no modelo de efeito-fixo. 
Considerando-se as principais variáveis incluídas no modelo, um aumento dos anos médios de educação feminina é a variável explicativa que ocasiona o maior impacto na redução da fecundidade.

Da mesma forma, a taxa de participação feminina no mercado de trabalho possui um efeito negativo e significante na variável dependente, como é esperado pelo modelo. $\mathrm{O}$ aumento de dez por cento na participação feminina reduz aproximadamente 0,5 filhos por mulher.

Anos médios de educação masculina possui um efeito absoluto menor na variável dependente e positivo, após o controle do efeito-fixo por país. Como previsto teoricamente, quando o tempo que os pais gastam no cuidado dos filhos não os impede de participar no mercado de trabalho, o aumento dos anos de estudo masculino estaria associado ao aumento da produtividade, renda do trabalho e recursos disponíveis para o cuidado dos filhos. Portanto, teria um impacto positivo na fecundidade.

Corroborando expectativas teóricas, um aumento da mortalidade infantil leva a um efeito reposição dos filhos mortos pelos pais, aumentando as taxas de fecundidade. $\mathrm{O}$ aumento da mortalidade infantil em 10 crianças para cada mil pessoas aumenta a fecundidade em 0,1 .

A variável expectativa de vida, componente do IDH, não possui impacto estatisticamente significante. Isso pode ser explicado devido a sua alta correlação negativa ($0,94)$ com a variável mortalidade infantil, o que dificulta a determinação do impacto das duas variáveis separadamente.

O PIB per capita tem um impacto significantemente nulo, ou seja, não altera a demanda por filhos. Como conclusão, ressalta-se a importância de melhorias no acesso e qualidade dos serviços de saúde, bem como do nível de educação feminina, como formas de controle eficaz da fecundidade.

Devido a possível presença de endogeneidade nas estimativas apresentadas no modelo estático, apresentamos também as estimativas pelo método dos momentos generalizados de Arellano-Bond. Através desse método, podemos estimar um painel dinâmico e encontrar instrumentos, não somente para a variável dependente defasada endógena, mas também para as demais variáveis explicativas do modelo. Os resultados estão descritos na Figura 3.

O modelo dinâmico inclui as defasagens da variável dependente como variáveis explicativas. A inclusão dessas variáveis dependentes defasadas permite controlar pela possível omissão de variáveis explicativas e combater o viés de má especificação do modelo.

Foram incluídas duas defasagens da variável dependente, visto que o teste de autocorrelação residual mostrou evidência de autocorrelação de segunda-ordem para o 
modelo que inclui apenas uma defasagem da variável dependente. De acordo com Arellano e Bond (1991), autocorrelação de segunda ordem implicaria em estimativas inconsistentes para os parâmetros do modelo. O modelo com duas defasagens não apresenta evidência de autocorrelação de segunda ordem.

Os principais resultados encontrados no modelo estático permanecem válidos. A variável relativa a anos médios de educação feminina possui o maior impacto absoluto na taxa de fecundidade dentre as variáveis explicativas, não incluindo nesse conjunto a variável dependente defasada. $\mathrm{O}$ aumento de um ano de educação feminina ocasiona uma queda de aproximadamente 0,4 filhos por mulher.

Esse estudo aponta que a associação negativa entre índice de desenvolvimento humano e fecundidade deve ser atribuída aos componentes de saúde e, principalmente, de educação presentes no índice. De acordo com o modelo estimado, o aumento da educação feminina ocasiona uma queda significativa no número de nascidos por mulher, sendo a principal variável explicativa da fecundidade. Nossos resultados encontram respaldo na literatura acadêmica como evidenciado por Cygan-Rehm e Maeder (2013).

\section{Considerações finais}

Para reduzir o crescimento populacional, alguns países adotam políticas públicas severamente criticadas pela opinião pública. Nas décadas de 1970 e 1980, diversos grupos feministas e defensores dos direitos reprodutivos lutavam contra as políticas de controle populacional compulsório que alguns países adotavam para limitar o tamanho das famílias (UNFPA, 2019).

A Conferência Internacional sobre População e Desenvolvimento das Nações Unidas realizada em 1994 colocou, pela primeira vez, a necessidade de dar direito aos casais de escolher o número desejado de filhos e o momento mais oportuno para as suas concepções. Seria necessário dar acesso a métodos de planejamento familiar, informar os indivíduos e aumentar a igualdade de gênero nas escolhas sexuais e reprodutivas.

Empiricamente, Bryant (2007) e Araújo Junior, Salvato e Queiroz (2013) apresentam evidência de que um maior desenvolvimento socioeconômico estaria associado a menores níveis de fecundidade. No presente estudo, analisamos as associações entre a fecundidade e as diferentes dimensões socioeconômicas: nível de educação, saúde e renda de uma população.

Para isso, revisamos os modelos teóricos de demanda por filhos e avaliamos a contrapartida empírica desses modelos em uma base de dados internacionais. Controlando-se 
pelos demais fatores, a variável anos-médios de educação feminina possui o maior impacto absoluto na taxa de fecundidade. $\mathrm{O}$ aumento de um ano médio de educação feminina ocasiona uma queda de 0,6 filhos por mulher no modelo estático e de 0,4 filhos por mulher no modelo dinâmico. Destaca-se também a associação positiva entre fecundidade e a mortalidade infantil. Entretanto, a variável renda não apresenta associação com a fecundidade, após o controle das métricas de saúde e educação.

Os resultados abrem espaço para a discussão de políticas públicas que considerem o impacto da educação feminina no dimensionamento populacional. $\mathrm{O}$ aumento da escolaridade feminina está possivelmente associado a um melhor entendimento e escolha mais adequada de métodos de planejamento familiar, além do empoderamento feminino na defesa de suas escolhas reprodutivas. Ademais, visto que o aumento da educação feminina implica em ganhos de produtividade no mercado de trabalho, aumentos salariais e acesso a melhores cuidados pré-natais, cabe aos estudos vindouros analisar se o aumento da educação feminina impacta também a saúde e a qualidade de vida dos recém-nascidos.

\section{REFERÊNCIAS}

ARAÚJO JUNIOR, A. F.; SALVATO, M.; QUEIROZ, B. L. Desenvolvimento e Fecundidade no Brasil: Reversão da Fecundidade para Municípios mais Desenvolvidos. Planejamento e Políticas Públicas, n. 41, jul./dez. 2013.

ARELLANO, M. Panel data econometrics. Oxford University Press, 2003. 231 p.

BARRO, R. J.; LEE, J. W. A new data set of educational attainment in the world, 1950-2010. Journal of development economics, v. 104, p. 184-198, 2013.

BECKER, G. S. An economic analysis of fertility. In: Demographic and economic change in developed countries. Columbia University Press, 1960. p. 209-240.

BERQUÓ, E. S.; CAVENAGHI, S. M. Notas sobre os diferenciais educacionais e econômicos da fecundidade no Brasil. Revista Brasileira de Estudos de População, v. 31, n. 2, p. 471-482, 2014.

BLAKE, J. Family size and the quality of children. Demography, v. 18, n. 4, p. 421-442, 1981.

BRANDÃO, E. R. Gravidez na adolescência: um balanço bibliográfico. In: HEILBORN M. L.; AQUINO E. M. L.; KNAUTH D. R.; BOZON M. (Org.). O aprendizado da sexualidade: reprodução e trajetórias sociais de jovens brasileiros. Rio de Janeiro: FIOCRUZ, 2006. p. 61-95. 
BRYANT, J. Theories of fertility declines and the evidence from development indicators. Population and development review, v. 33, n. 1, p. 101-127, 2007.

CEPI-DSS/ENSP/FIOCRUZ. Taxa de fecundidade total, por ano, segundo escolaridade [online]. Rio de Janeiro: Portal Determinantes Sociais da Saúde. Observatório sobre Iniquidades em Saúde, 2012. Disponível em: http://dssbr.org/site/wpcontent/uploads/2012/03/Ind010106-20120130.pdf. Acesso em: 15 ago. 2020.

CYGAN-REHM, K.; MAEDER, M. The effect of education on fertility: evidence from a compulsory schooling reform. Labour Economics, v. 25, p. 35-48, 2013.

MUNDIAL, B. World Development Indicators, 2015.

SCHULTZ, T. P. Demand for children in low income countries. Handbook of population and family economics, v. 1, p. 349-430, 1997.

UNFPA (United Nations Population Fund). State of World Population 2019. Unfinished Business: the pursuit of rights and choices for all. New York: UNFPA, 2019.

ZHANG, J. The evolution of China's one-child policy and its effects on family outcomes. The Journal of Economic Perspectives, v. 31, n. 1, p. 141-159, 2017.

\section{Como referenciar este artigo}

POIATTI, N. D. Escolaridade feminina e a sua importância na escolha voluntária da fecundidade. Revista Ibero-Americana de Estudos em Educação, Araraquara, v. 15, n. 4, p. 1786-1798, $\quad$ out./dez. $2020 . \quad$ e-ISSN: $1982-5587 . \quad$ DOI: https://doi.org/10.21723/riaee.v15i4.13182

Submetido em: 09/12/2019

Revisões requeridas em: $25 / 02 / 2020$

Aprovado em: 30/04/2020

Publicado em: 30/08/2020 
RIAEE - Revista Ibero-Americana de Estudos em Educação Araraquara, v. 15, n. 4, p. 1786-1798, out./dez. 2020. e-ISSN: $1982-5587$. DOI: https://doi.org/10.21723/riaee.v15i4.13182 